\title{
Impact of Confinement on the Burden of Caregivers of Patients with the Behavioral Variant of Frontotemporal Dementia and Alzheimer Disease during the COVID-19 Crisis in France
}

\author{
Claire Boutoleau-Bretonnière ${ }^{a, b}$ Hélène Pouclet-Courtemanche ${ }^{a, b}$ \\ Aurélie Gillet ${ }^{a} \quad$ Amélie Bernard $^{a, b}$ Anne-Laure Deruet ${ }^{a, b}$ \\ Inès Gouraud ${ }^{a, b}$ Estelle Lamy ${ }^{a, b} \quad$ Aurélien Mazoué $^{b} \quad$ Laëtitia Rocher $^{a}$ b, c \\ Cédric Bretonnière ${ }^{a}$ Mohamad El Haj ${ }^{c} d, e$

\begin{abstract}
${ }^{a} \mathrm{CHU}$ Nantes, Inserm ClC04, Nantes, France; bervice de Neurologie, Centre Mémoire de Ressources et Recherche, CHU Nantes, Nantes, France; ' Laboratoire de Psychologie des Pays de la Loire (LPPL - EA 4638), Universités d'Angers et Nantes, Nantes, France; ${ }^{\mathrm{d} U n i t e ́ ~ d e ~ G e ́ r i a t r i e, ~ C e n t r e ~ H o s p i t a l i e r ~ d e ~ T o u r c o i n g, ~ T o u r c o i n g, ~ F r a n c e ; ~}$
\end{abstract} \\ e Institut Universitaire de France, Paris, France
}

\section{Keywords}

Alzheimer disease - Behavioral disorders - Behavioral variant of frontotemporal dementia .

Confinement $\cdot$ COVID-19

\begin{abstract}
Introduction: The clinical presentation of the behavioral variant of frontotemporal dementia (bvFTD) differs from that of Alzheimer disease (AD), with major impairments in behavioral functions in bvFTD and cognitive impairment in AD. Both behavioral disturbances in bvFTD and cognitive impairment in AD contribute to caregiver burden. Objective: To investigate the impact of home confinement during the COVID-19 crisis on the burden of caregivers of bvFTD or AD patients. Methods: During the COVID-19 lockdown in France, neurologists and neuropsychologists from the Memory Center of Nantes Hospital conducted teleconsultations for 38 AD patients and 38 bvFTD patients as well as for their caregivers. During these consultations, caregivers were invited to rate the change in their burden during home confinement. They were also invited to rate behavioral or emotional changes in the patients during, compared with before, the confinement. Results: Twenty-two bvFTD caregivers and 14 AD caregivers experienced an increase in burden. For bvFTD caregivers, this increased burden occurred regardless of behavioral changes, while AD caregivers experienced an increased
\end{abstract}


burden related to changes in patients' neuropsychiatric symptoms. Among the whole cohort, 2 factors were associated with increased caregiver burden: behavioral change and bvFTD. Conclusion: The results demonstrate that during home confinement in the COVID-19 crisis, neuropsychiatric symptoms were the core factor that impacted caregiver burden in different ways depending on the disease.

(C) 2020 The Author(s)

Published by S. Karger AG, Basel

\section{Introduction}

The clinical presentation of frontotemporal dementia (FTD) is distinct from that of Alzheimer disease (AD). Indeed, there is a major impairment in executive and behavioral functions in FTD as opposed to cognitive impairment in AD. Patients with the behavioral variant of FTD (bvFTD) show early changes in personality and social conduct. Increased neuropsychiatric symptoms are associated with overall caregiver burden. It is now well known that this burden is correlated with the patient's level of behavioral disturbance and autonomy $[1,2]$. However, it also depends on the experience and personality of the caregiver [3]. In this study, we assessed the burden of caregivers of patients with bvFTD or AD. More specifically, we investigated this burden during home confinement in the COVID-19 pandemic. To cope with the infection and to limit its spread, people in France and Europe in general were confined to their homes and were only permitted to leave home to buy essential goods, briefly go out to exercise, or visit doctors. During the lockdown, the social distancing measures and the diminished physical contact with family and the outside world might be expected to significantly increase loneliness and impact the mental health of patients with AD or bvFTD and their caregivers. During this period, social activities (day care, cognitive stimulation) or even well-being activities were no longer possible.

To investigate whether social and physical restrictions during the COVID-19 crisis affected the caregivers of patients with FTD and AD, we evaluated the burden of caregivers who were confined to home with patients during the COVID-19 quarantine in France. Our study was based on a teleconsultation designed by the Memory Center of Nantes University Hospital. More specifically, during the lockdown, we contacted by phone caregivers and patients followed by the Memory Center of Nantes. The aim of our study was to assess neuropsychiatric functioning in the patients and the burden of their caregivers.

\section{Methods}

\section{Participants}

Data were collected from caregivers of patients with bvFTD and AD, recruited from the Memory Center of Nantes University Hospital between March and May 2020. Caregivers were defined as persons providing unpaid care for a spouse, relative, or friend.

Diagnosis had been made prior to the study by neurologists at the Memory Center. Probable AD was diagnosed according to the clinical criteria of the National Institute on Aging and the Alzheimer's Association criteria [4]. Probable FTD was diagnosed according to the current criteria for behavioral FTD [5]. This study was designed and conducted in accordance with the Declaration of Helsinki. Caregivers and patients (or their legal guardians) have given their oral informed consent (the study was carried out by telephone). The study was approved by the Ethics Committee of GERIA (Groupe Ethique Recherche Institut Angers; approval No. 2022-001).

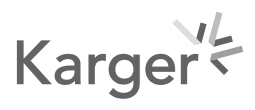




\section{Procedures}

During the confinement, between March 26, and May 9, 2020, neurologists and neuropsychologists from the Memory Center of Nantes conducted teleconsultations. During these consultations, standardized surveys were performed. Caregivers were invited to provide the exact date at which they were confined to their homes. They were invited to describe their experience during the confinement. They were also asked if they had noticed any behavioral or emotional changes in the patients during, compared with before, the confinement. If the answer was "yes," caregivers were invited to answer the Neuropsychiatric Inventory (NPI) questionnaire [6]. We used this questionnaire because it is based on interviewing caregivers, and it was possible to administer it over the phone during the lockdown. We asked caregivers to rate the severity of 12 symptoms: delusions, hallucinations, agitation, depression, anxiety, euphoria, apathy, disinhibition, irritability, aberrant motor activity, nighttime behavior disturbance, and appetite disorders. Severity was rated on a 3-point scale (1 point = mild, i.e., noticeable, but not a significant change; 2 points = moderate, i.e., significant, but not a dramatic change; and 3 points = severe, i.e., very marked or prominent, a dramatic change). Caregivers were also invited to rate the distress they experienced due to each symptom on a 5-point scale ( 0 = not distressing at all; 1 = minimal, i.e., slightly distressing, not difficult to cope with; $2=$ mild, i.e., not very distressing, generally easy to cope with; $3=$ moderate, i.e., fairly distressing, not always easy to cope with; $4=$ severe, i.e., very distressing, difficult to cope with; and $5=$ very severe, i.e., extremely distressing, impossible to cope with.

We also invited caregivers to provide information about their burden through the question: "Did your burden increase during confinement?" If the response was yes, caregivers were invited to rate whether this increase occurred: 1 = rarely; 2 = sometimes; 3 = quite frequently; and 4 = nearly always. We also asked them if the increased burden was linked to: (1) behavioral modification; (2) behavioral disorders without modification during quarantine; (3) stopping of the patient's activities; (4) interruption of the caregiver's own activities; (5) stopping of home care assistance; and (6) other factors.

\section{Statistical Analysis}

Results were expressed as means \pm standard deviations or medians with interquartile ranges. Categorical data were presented as percentages and were compared using Fisher's exact test. Continuous data were compared using nonparametric tests. Missing data were treated using the simplest approach: analyses were performed only on the completed cases. Missing data were excluded from the analysis.

The main outcome measure was caregiver burden. To investigate the link between different variables and caregiver burden, a binary logistic regression model was used. Variables with a value of $p<0.20$ in univariate analysis were entered into the models. The final model was kept because of its ability to best predict the outcome, maximizing the likelihood ratio. The highest Nagelkerke $R^{2}$ then obtained is presented with the final model.

Analyses with a value of $p<0.05$ were considered statistically significant. The analysis was carried out using IBM SPSS Statistics version 19 software (IBM, Armonk, NY, USA).

\section{Results}

Between March 26 and May 9, 2020, 128 telephone consultations were performed. These consultations concerned 38 patients (23 men and 15 women) fulfilling NINCDS-ADRDA criteria for probable AD [4] and 38 patients (22 men and 16 women) fulfilling Rascovsky's criteria for the behavioral variant of FTD [5]. Both groups were not significantly different in terms of age $(71.9 \pm 8.2$ vs. $67.8 \pm 9.0$ years), MMSE score (19.4 \pm 6.2 vs. $18.4 \pm 7.8$ ) or esti-

\section{Karger'}


Table 1. Factors that could influence caregiver burden in the AD group

\begin{tabular}{|c|c|c|c|c|}
\hline & & $\begin{array}{l}\text { No change in burden } \\
n=24\end{array}$ & $\begin{array}{l}\text { Change in burden } \\
n=14\end{array}$ & $p$ \\
\hline Time of call & (Duration of confinement, days) & $25.5 \pm 15.6$ & $30.5 \pm 12.2$ & 0.186 \\
\hline \multirow[t]{4}{*}{ Patient } & Age, years & $69.3 \pm 6.3$ & $75.1 \pm 10$ & 0.04 \\
\hline & Sex, females/males & 13/11 & $10 / 4$ & NS \\
\hline & Disease duration, years & $4.7 \pm 2$ & $5.5 \pm 2.4$ & NS \\
\hline & $\operatorname{MMSE}(/ 30)$ & $21 \pm 5.4$ & $16.5 \pm 6.7$ & 0.04 \\
\hline \multirow[t]{4}{*}{ Caregiver } & Age, years & $65.7 \pm 10.4$ & $73.9 \pm 14.3$ & 0.11 \\
\hline & Sex, females/males & $15 / 9$ & $7 / 7$ & NS \\
\hline & Type, spouse/other & $19 / 6$ & $11 / 3$ & NS \\
\hline & Live in the same house, yes/no & $18 / 3$ & $13 / 1$ & NS \\
\hline Change in behavior & Yes/no & $0 / 24$ & $10 / 4$ & $<10^{-5}$ \\
\hline
\end{tabular}

Means \pm SD or proportions. NS, nonsignificant. Values of $p<0.2$ are detailed (nonparametric tests such as the Mann-Whitney or Fisher exact test). Only change in behavior was significantly different $(p<0.05)$.

mated duration of disease ( $5.1 \pm 2.6$ vs. $4.2 \pm 3.1$ years). The AD caregiver group comprised 14 men and 24 women; 30 were spouses and 31 lived in the same house as the patient. The FTD caregivers group comprised 16 men and 22 women; 34 were spouses and 33 lived in the same house as the patient. There were significant differences between both caregiver groups with respect to age (AD caregivers: mean $68.4 \pm 12.2$ years/bvFTD caregivers: mean $59.8 \pm$ 11.1 years, $p=0.023)$. No difference was found for the duration of confinement at time of call (AD group: $27.4 \pm 14.5$ days vs. bvFTD group: $28.1 \pm 10.2$ days).

As expected, a higher proportion of caregivers of bvFTD patients experienced an increase in burden when compared to caregivers of $\mathrm{AD}$ patients but without reaching statistical difference (58\% FTD caregivers vs. 37\% AD caregivers).

The score of burden for caregivers who experienced a change in burden was not different between both groups (AD caregivers $2.43 \pm 1.2$ vs. FTD caregivers $2.40 \pm 1.9$ ). Responses of caregivers concerning factors associated with the increase in burden were: behavioral modification reported by 9 AD caregivers versus 5 bvFTD caregivers; behavioral disorders without modification during quarantine reported by 4 AD caregivers versus 16 bvFTD caregivers; interruption of patient's activities (cognitive stimulation, day center etc.) reported by $5 \mathrm{AD}$ caregivers versus 4 bvFTD caregivers; interruption of caregiver's own activities reported by 1 AD caregiver versus 6 bvFTD caregivers; and interruption of home care assistance reported by 1 AD caregiver versus 7 bvfTD caregivers. There were no significant differences between both groups.

During confinement, 8 patients with bvFTD presented neuropsychiatric changes, and 10 patients with $\mathrm{AD}$ experienced changes. There were no differences between both groups for NPI severity (AD patients: $5.2 \pm 4.92$ vs. FTD patients: $2.9 \pm 1.7$ ) or for NPI distress (AD patients $5.1 \pm 4.99$ vs. FTD patients $3.1 \pm 2.0$ ). bvFTD patients had the highest score for apathy $(1.5 \pm 1.1)$, and $A D$ patients had the highest score for agitation $(1.0 \pm 1.7)$.

We conducted univariate analysis to understand which factors could influence caregiver burden. The results for AD caregivers are reported in Table 1 and the results for bvFTD caregivers in Table 2. The analysis revealed that the burden of AD caregivers increased when changes in patient behavior occurred during confinement; caregiver burden increased when patients were oldest, and when MMSE scores were lower. For the bvFTD caregiver group, no such association was found. 
Table 2. Factors that could influence caregiver burden in the bvFTD group

\begin{tabular}{llcc}
\hline & & $\begin{array}{l}\text { No change in burden } \\
n=16\end{array}$ & $\begin{array}{l}\text { Change in burden } \\
n=22\end{array}$ \\
\hline \multirow{2}{*}{ Time of call } & (Duration of confinement, days) & $27.3 \pm 10.2$ & $28.7 \pm 10.4$ \\
\hline Patient & Age, years & $67.7 \pm 9.1$ & $67.8 \pm 9.2$ \\
& Sex, females/males & $5 / 11$ & $11 / 11$ \\
& Duration of disease, years & $5.1 \pm 3.6$ & $4.1 \pm 2.8$ \\
& MMSE (/30) & $16.8 \pm 8.2$ & $19.7 \pm 7.3$ \\
\hline Caregiver & Age, years & $58.5 \pm 6.3$ & $60.1 \pm 13.3$ \\
& Sex, females/males & $11 / 5$ & $13 / 9$ \\
& Type, spouse/other & $14 / 2$ & $20 / 2$ \\
& Live in the same house, yes/not & $14 / 2$ & $19 / 3$ \\
\hline Change in behavior & Yes/no & $2 / 14$ & $6 / 16$ \\
\hline
\end{tabular}

Means \pm SD or proportions. No significant difference was found (Mann-Whitney or Fisher exact test).

Table 3. Factors associated with caregiver burden in a cohort of 76 dyads

\begin{tabular}{lclrl}
\hline & $\exp (\mathrm{B}) \mathrm{OR}$ & $95 \% \mathrm{CI}$ & \multicolumn{1}{l}{$p$} \\
\hline Change in behavior & 20.0 & 3.7 & 108.1 & 0.001 \\
Disease (FTD vs. AD) & 3.2 & 1.0 & 10.0 & 0.042 \\
Caregiver (spouse or other) & 1.8 & 0.3 & 9.6 & $\mathrm{NS}$ \\
Duration of quarantine $(>25$ days) & 1.7 & 0.6 & 5.2 & $\mathrm{NS}$ \\
\hline
\end{tabular}

The presented final model had 76 observations entered and yielded the highest likelihood ratio. Nagelkerke $R^{2}=0.38$. The 4 presented variables were those included in the final logistic model. Values of $p<0.2$ are detailed. Significant differences $(p<0.05)$ are italicized.

We also conducted a binary regression analysis concerning all caregivers in order to analyze the factors that influenced caregiver burden. Change in behavior and being a caregiver of a bvFTD patient were the factors correlated with increased caregiver burden (Table 3).

\section{Discussion}

Among our cohort of 38 patients with bvFTD, only 8 demonstrated neuropsychiatric changes during the confinement. Ten out of $38 \mathrm{AD}$ patients also experienced neuropsychiatric changes. More caregivers of bvFTD patients than caregivers of AD patients were affected by the confinement. Patient behavior, with or without changes, increased bvFTD caregiver burden, while behavior changes were the principal factor that impacted AD caregivers.

Responsibility for the daily care of patients with cognitive impairment living at home is often assumed by the spouse or children. This care has important repercussions for the caregivers' physical and psychological well-being. Many caregivers thus present symptoms of exhaustion, stress, and depression [7]. The studies of Andrieu et al. [8] and Hebert et al. [1] showed that the burden is independently influenced by the extent of the behavioral disorder. Previous studies have also documented the heavy burden imposed by bvFTD on caregivers 
[2, 9-11]. bvFTD was associated with a substantial modification in the life of dyads, including diminished quality of life and increased caregiver burden. This research highlights how bvFTD impacted the caregivers' well-being. In our study, many caregivers reported during the telephone consultation that their spouses had been "confined" since the onset of the disease because of apathy, as demonstrated by our results showing that the score for apathy on the NPI was higher for bvFTD patients than for AD patients. For bvFTD caregivers, this increased burden occurred regardless of behavioral changes since behavioral disorders were already hard to manage before the pandemic. During the lockdown, they were left alone and confronted with an increase in neuropsychiatric symptoms.

Interruption of external activities for the caregiver and interruption of home care assistance were relevant factors explaining the increased burden for bvFTD caregivers. In a study comparing the intensity of the burden between AD and FTD using the NPI-assessed caregiver impact, de Vugt et al. [3] showed that apathy was very stressful for the caregiver. Apathy, defined as a decrease in motivation, leads to social isolation. As a result, this symptom, a priori not very striking, is very often very badly tolerated by the patient's entourage [12]. It was precisely the leading symptom in terms of bvFTD patients' NPI score in our study.

Conversely, the increased caregiver burden of AD patients during confinement was largely associated with changes in patient behavior such as agitation. These changes were correlated with old age and an advanced stage of the disease. The results of our study are in accordance with our knowledge of both diseases: in bvFTD, behavioral disorders start at the beginning of the disease whereas in AD positive symptoms usually occur later. Numerous studies have shown that symptoms like agitation and irritability are very distressing for caregivers $[13,14]$.

In our study, we did not demonstrate differences between $\mathrm{AD}$ and bvFTD patients in terms of their NPI scores. However, these scores only reflect behavioral disorders of patients who experienced changes in behavior. Our goal was to highlight the change in behavior, so we took care of questioning all caregivers on these symptoms but did perform the NPI only when a change had occurred. It is also now well known that NPI is not the most appropriate scale to use for bvFTD patients. The frontal behavioral inventory [15], the frontotemporal behavioral scale [16] and, more recently, DAPHNE [17] are more specific for frontal behavioral disorders. Other potential limitations of our study include the lack of caregiver burden assessment and the lack of a neuropsychiatric assessment before the confinement. Note, however, that the confinement was somewhat unpredictable, which means we were unable to conduct an assessment before study initiation.

Despite its potential limitations, this study has implications for clinicians. Our results highlight the importance of supporting caregivers during crises, such as the COVID-19 pandemic, to help them cope with their own social isolation and with the neuropsychiatric disorders observed in the patients in their care. It has also been shown by Sink et al. [18] that certain characteristics of caregivers are associated with behavioral disorders, regardless of the characteristics of the patients. Thus, spending a lot of time with the demented person was associated with an increase in the number of reported behavioral disorders. Quarantine has by definition forced caregivers to be with their loved ones all the time without being able to escape. There are many methods of psychological support for carers: psycho-educational programs, discussion groups, and individual psychotherapy, for example. The most effective methods seem to be either individual sessions to learn techniques that will enable carers to cope with a patient's behavioral disorders, or sessions with pairs aimed at helping the caregiver in setting up effective coping strategies.

Continuation of personal leisure and social exchanges are important for coping with both diseases. The management of AD or FTD patients must, therefore, imperatively take the patient-caregiver dyad into account.

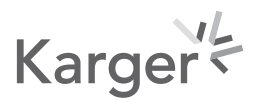




\section{Disorders Extra}

\section{Conclusion}

This is the first study on the caregiver burden during the COVID-19 crisis. Our study demonstrates how behavioral disorders during the confinement can increase the burden for caregivers of FTD patients and caregivers of AD patients, although not in the same manner. Our findings also highlight the impact of social isolation on caregiver burden and the importance of differentiating between diagnostic groups and specific behavioral domains when focusing on caregiver burden.

\section{Acknowledgment}

The authors would like to thank caregivers and patients.

\section{Statement of Ethics}

The research was conducted ethically in accordance with the World Medical Association Declaration of Helsinki. Caregivers and patients (or their legal guardians) have given their oral informed consent (the study was carried out by telephone). The study was approved by the Ethics Committee of GERIA (Groupe Ethique Recherche Institut Angers; approval No. 2022-001).

\section{Conflict of Interest Statement}

The authors have no conflicts of interest to declare.

\section{Funding Sources}

None.

\section{Author Contributions}

C.B.-B. and H.P.-C.: conception and design of the work, the acquisition, analysis and interpretation of data for the work; final approval of the version to be published; accountable for all aspects of the work in ensuring that questions related to the accuracy or integrity of any part of the work are appropriately investigated and resolved. C.B.-B.: drafting the work; H.P.-C: revising the work; A.G., A.B., A.-L.D., I.G., E.L., A.M., L.R., C.B, and M.E.H.: acquisition, analysis and interpretation of data for the work; revising the work; final approval of the version to be published; accountable for all aspects of the work in ensuring that questions related to the accuracy or integrity of any part of the work are appropriately investigated and resolved.

\section{Karger'k}




\section{References}

1 Hebert R, Bravo, G, Preville M. Reliability, Validity and Reference Values of the Zarit Burden Interview for Assessing Informal Caregivers of Community-Dwelling Older Persons with Dementia. Can J Aging 2000;19: 494-507.

2 Boutoleau-Bretonnière C, Vercelletto M, Volteau C, Renou P, Lamy E. Zarit burden inventory and activities of daily living in the behavioral variant of frontotemporal dementia. Dement Geriatr Cogn Disord. 2008;25(3): 272-7.

3 de Vugt ME, Stevens F, Aalten P, Lousberg R, Jaspers N, Winkens I, et al. Do caregiver management strategies influence patient behaviour in dementia? Int J Geriatr Psychiatry. 2004 Jan;19(1):85-92.

4 McKhann GM, Knopman DS, Chertkow H, Hyman BT, Jack CR Jr, Kawas CH, et al. The diagnosis of dementia due to Alzheimer's disease: recommendations from the National Institute on Aging-Alzheimer's Association workgroups on diagnostic guidelines for Alzheimer's disease. Alzheimers Dement. 2011 May;7(3):263-9.

5 Rascovsky K, Hodges JR, Knopman D, Mendez MF, Kramer JH, Neuhaus J, et al. Sensitivity of revised diagnostic criteria for the behavioural variant of frontotemporal dementia. Brain. 2011 Sep;134(Pt 9):2456-77.

6 Cummings JL, Mega M, Gray K, Rosenberg-Thompson S, Carusi DA, Gornbein J. The Neuropsychiatric Inventory: comprehensive assessment of psychopathology in dementia. Neurology. 1994 Dec;44(12):2308-14.

7 Pinquart M, Sörensen S. Differences between caregivers and noncaregivers in psychological health and physical health: a meta-analysis. Psychol Aging. 2003 Jun;18(2):250-67.

8 Andrieu S, Balardy L, Gillette-Guyonnet S, Bocquet H, Cantet C, Albarède JL, et al. [Burden experienced by informal caregivers assisting Alzheimer's patients in the REAL.FR study]. Rev Med Interne. 2003 Oct;24 Suppl 3:351s-9s.

9 Diehl-Schmid J, Schmidt EM, Nunnemann S, Riedl L, Kurz A, Förstl H, et al. Caregiver burden and needs in frontotemporal dementia. J Geriatr Psychiatry Neurol. 2013 Dec;26(4):221-9.

10 Mioshi E, Foxe D, Leslie F, Savage S, Hsieh S, Miller L, et al. The impact of dementia severity on caregiver burden in frontotemporal dementia and Alzheimer disease. Alzheimer Dis Assoc Disord. 2013 Jan-Mar;27(1):68-73.

11 Riedijk SR, De Vugt ME, Duivenvoorden HJ, Niermeijer MF, Van Swieten JC, Verhey FR, et al. Caregiver burden, health-related quality of life and coping in dementia caregivers: a comparison of frontotemporal dementia and Alzheimer's disease. Dement Geriatr Cogn Disord. 2006;22(5-6):405-12.

12 Truzzi A, Valente L, Engelhardt E, Laks J. The association between caregiver distress and individual neuropsychiatric symptoms of dementia. Dement Neuropsychol. 2013 Jul-Sep;7(3):286-91.

13 Fauth EB, Gibbons A. Which behavioral and psychological symptoms of dementia are the most problematic? Variability by prevalence, intensity, distress ratings, and associations with caregiver depressive symptoms. Int J Geriatr Psychiatry. 2014 Mar;29(3):263-71.

14 Feast A, Moniz-Cook E, Stoner C, Charlesworth G, Orrell M. A systematic review of the relationship between behavioral and psychological symptoms (BPSD) and caregiver well-being. Int Psychogeriatr. 2016 Nov; 28(11):1761-74.

15 Kertesz A, Davidson W, Fox H. Frontal behavioral inventory: diagnostic criteria for frontal lobe dementia. Can J Neurol Sci. 1997 Feb;24(1):29-36.

16 Lebert F, Pasquier F, Souliez L, Petit H. Frontotemporal behavioral scale. Alzheimer Dis Assoc Disord. 1998 Dec;12(4):335-9.

17 Boutoleau-Bretonnière C, Evrard C, Hardouin JB, Rocher L, Charriau T, Etcharry-Bouyx F, et al. DAPHNE: A New Tool for the Assessment of the Behavioral Variant of Frontotemporal Dementia. Dement Geriatr Cogn Disord Extra. 2015 Dec;5(3):503-16.

18 Sink KM, Covinsky KE, Barnes DE, Newcomer RJ, Yaffe K. Caregiver characteristics are associated with neuropsychiatric symptoms of dementia. J Am Geriatr Soc. 2006 May;54(5):796-803. 